\title{
A PARTICIPAÇÃO FEMININA NOS CURSOS DE COMPUTAÇÃO DO ESTADO DO PARÁ: AVANÇOS E DESAFIOS
}

\section{FEMININE PARTICIPATION IN COMPUTATION COURSES IN THE STATE OF PARÁ: ADVANCES AND CHALLENGES}

\author{
Mônica Sousa da Silva ${ }^{1}$, Aleksandra do Socorro da Silva ${ }^{1}$, Silvana Rossy \\ de Brito ${ }^{1}$ \\ ${ }^{1}$ Instituto Ciberespacial - ICIBE, Universidade Federal Rural da Amazônia - \\ UFRA \\ E-mail: demonicasilva@gmail.com, aleksandra.silva@ufra.edu.br, \\ silvana.rossy@ufra.edu.br
}

RESUMO - Este artigo, através de questionários estruturados, realiza uma análise das respostas dos(as) discentes com respeito à diferentes aspectos, sob a ótica do gênero do discente: motivos para a seleção do curso, nível de satisfação e desmotivação com o curso, influência dos grupos de disciplinas que levaram à desmotivação, dificuldade de aprendizado com as disciplinas de algoritmos e programação, motivos para permanecer no curso, dentre outros. A metodologia envolve três etapas: análise das respostas dos(as) discentes nos questionários; estudo da associação entre as respostas dos(as) discentes; e, entrevistas com discentes. Como resultados, foram encontradas participações significativamente menores das mulheres nos cursos analisados e associação significativa entre inibição e dificuldade de aprendizado relatadas. Embora o estudo aponte que há pouca influência do sexo do(a) discente nos aspectos de inibição, dificuldade de aprendizado e desmotivação, os relatos dos estudantes apontam para a necessidade de iniciativas, programas e ações que incentivam a participação feminina nesses cursos.

Palavras-chave: mulheres; empoderamento; tecnologia da informação.

Recebido em: 10/08/2016 Revisado em: 30/11/2016 Aprovado em: 07/02/2017
ABSTRACT - This article, through structured questionnaires, performs an analysis of the students' responses with respect to different aspects, from the viewpoint of the student's gender: reasons for selection the course, level of satisfaction and demotivation with the course, influence of the groups of disciplines for demotivation, learning difficulties with disciplines of algorithm and programming, reasons to stay in the course, among others. The methodology involves three steps: analysis of the students' answers in the questionnaires; study of the association between the students' answers; And, interviews with women. The results showed a significantly lower participation of women in the analyzed courses and a significant association between inhibition and learning difficulties. Although the study indicates that there a low influence of students' sex in the aspects of inhibition, learning difficulties and demotivation, the findings, based on the student's narratives pointed to the need for initiatives, programs and actions that encourage the participation of women in these courses.

Keywords: women; empowerment; information technology. 


\section{INTRODUÇÃO}

A participação feminina na área da computação e informática ${ }^{1}$ tem sido alvo de discussões em diversos espaços nacionais e internacionais (SABOYA, 2013; SCHWARTZ et al., 2006), que buscam incentivar e impulsionar a participação das mulheres, contribuindo para reflexão acerca das relações de gênero, tanto na área de formação profissional, quanto de atuação dos profissionais de informática. Muitos desses trabalhos buscam mobilizar e efetivar políticas públicas para combater as profundas desigualdades de gênero e assim desconstruir os estereótipos que tanto afetam as mulheres, incentivando uma participação mais igualitária quanto à questão de gênero nessa área.

Em meio ao debate sobre a participação feminina, um conjunto de questões-problema são elencados: Como tem sido esta inserção das mulheres nos cursos de formação em Tecnologia da Informação (TI)? Quais as dificuldades que as mulheres enfrentam nesta área?

Nessa direção, através destas
indagações e, posteriormente das

1 No Brasil, as áreas de Informática, Computação e Tecnologia da Informação são frequentemente utilizadas como sinônimos, embora a Computação e Tecnologia da Informação esteja melhor associada com as Ciências da Informação, enquanto a Computação está mais direcionadas para o desenvolvimento de hardware e software. Porém, neste trabalho, esses termos são utilizados como sinônimos. apresentações das dificuldades apontadas a partir dos cenários enfrentados por diversas mulheres que ainda se arriscam nesta área onde predomina a presença masculina, busca-se identificar e verificar os avanços ocorridos e as dificuldades que ainda estão presentes para as mulheres que optam por essa área. Tais desigualdades são observadas em vários níveis, como por exemplo, a falta de modelos femininos na área, inibição dentro de sala de aula causada pela baixa autoestima, inviabilidade na sua produção acadêmica, a falta de incentivo para que sigam uma carreira na área, entre outros aspectos.

Neste contexto, este artigo está situado no estudo de indicadores de participação feminina nos cursos de computação (Bacharelado em Sistemas de Informação, Licenciatura em Computação e Bacharelado em Ciência da Computação), especificamente em Universidades públicas do Estado do Pará. O objetivo é analisar as respostas dos(as) discentes com respeito à diferentes aspectos: motivos para a seleção do curso, nível de satisfação e desmotivação com o curso, influência dos grupos de disciplinas que levaram à desmotivação, dificuldade de aprendizado com as disciplinas de algoritmos e programação, motivos para permanecer no curso, dentre outros, sob a ótica do gênero do discente. 
Este artigo está estruturado em 6 seções: a seção 2 apresenta uma revisão da literatura sobre a participação feminina nos cursos e no mercado de $\mathrm{Tl}$, questões de gênero e projetos que visam ampliar a participação feminina na área; a seção 3 apresenta materiais e métodos da pesquisa; a seção 4 apresenta resultados e discussões; e, finalmente, a seção 5 apresenta as considerações finais do estudo.

\section{REVISÃO DA LITERATURA}

Dentro da área de Tecnologia da Informação, as mulheres permanecem em segundo plano, por mais que a história da computação esteja marcada pela presença e grandes contribuições de diversas mulheres como Ada Lovelace, Ruth Teitelbaum, Frances Spence, Betty Holberton, Grace Hopper e HedyLamarr, entre outras percussoras da área. Cabral e Bazzo (2005) fazem uma importante retrospectiva histórica citada no trecho a seguir:

“Historicamente, as
mulheres foram afastadas
do círculo criativo e líder
da produção científica e
tecnológica. Isso limitou
sua atuação fora da esfera
privada da casa e foi,
séculos após séculos,
evidenciado pela sua
ausência e condução em
carreiras comorísica,
química, biologia,
matemática, engenharia e
computação. Essas áreas
desenvolveram-ser ao
sabor der valores
considerados a como
historicamente

masculinos - certeza, eficiência, controle, ordem. (p. 4).

Dentre as justificativas para a baixa participação feminina na área de TI está a existência de estereótipos, tanto no Brasil quanto no mundo, que consideram essa área muito técnica e pouco atrativa para as mulheres (UNIÃO EUROPEIA, 2008; NUNES, 2012; NUNES et al., 2015; CAMPBELL; HERLIHY, 2016).

Outrossim, para Gil-Juarez et al. (2011), uma melhor compreensão da problemática de gênero em $\mathrm{TI}$ requer que se considere o contexto social e educativo vinculado à socialização de gênero. Para os autores, homens e mulheres interiorizam normas, valores e formas diferenciadas de agir, de acordo com a sociedade em que nascem e crescem e, portanto, aprendem formas distintas de se relacionarem com a tecnologia. Como consequência, a falta de interesse feminino pelas TIs pode ser justificada pela construção sociocultural da tecnologia vinculada a um contexto masculino.

Considerando a participação das mulheres nos cursos superiores, segundo Nunes (2012), o número de mulheres inscritas em graduações na área de TI é reduzido, seguido também de uma diminuição do número de mulheres presentes nos programas de pós-graduação e 
neste mercado de trabalho (NUNES et al., 2015). Conforme dados da Pesquisa Nacional por Amostra de Domicílios (IBGE, 2014), a participação das mulheres na área de TI representa cerca de $20 \%$ do total de profissionais dessa área que atuam no Brasil.

Para alguns autores (MORAIS; RAMOS, 2016; DOMÍNGUEZ et al., 2011; GILJUAREZ et al., 2011), há um fosso digital de gênero (do inglês: gender digital divide) que pode ser entendido como a diferença entre homens e mulheres ao utilizarem e acessarem as TIs, revelando que os homens, devido às tradições patriarcais, estão em posição privilegiada na Era do Conhecimento (DOMíNGUEZ et al., 2011). Segundo esses autores, o fosso digital de gênero se dá principalmente em relação ao uso, frequência de utilização e competências, bem como na baixa representação feminina nos setores ligados à área de $\mathrm{TI}$.

Na tentativa de ampliar a participação feminina na área e nos cursos de $\mathrm{Tl}$ e reduzir o fosso digital de gênero, existem importantes iniciativas, através de programas e projetos que buscam combater os estereótipos existentes quanto ao gênero e a tecnologia. Como exemplo, no Brasil a Sociedade Brasileira de Computação (SBC) mantém o Programa "Meninas Digitais" que tem dentre os objetivos divulgar a área de Computação para despertar o interesse de estudantes do ensino médio/tecnológico ou dos anos finais do ensino fundamental, para que conheçam melhor a área, motivando-as a seguir carreira na Computação (SBC, 2016; MACIEL; BIM, 2016). O Programa "Meninas Digitais" tem projetos espalhados em vários estados brasileiros.

\section{MATERIAIS E MÉTODOS}

Esta pesquisa caracteriza-se por ser exploratória, quantitativa e qualitativa. Inicialmente, como fonte de dados foram capturadas informações públicas do Sistema Integrado de Gestão de Atividades Acadêmicas (SIGAA) da Universidade Federal Rural da Amazônia (UFRA) e da Universidade Federal do Pará (UFPA) com respeito ao número de alunos matriculados nos cursos de Bacharelado em Sistemas de Informação (SI), Ciência da Computação (CC) e Licenciatura em Computação (LC) nos campi dos municípios de Belém, Castanhal, Cametá e Tucuruí (Figura 1), identificando os alunos do sexo masculino (azul) e feminino (vermelho). Nesta análise, 33\% dos participantes são do sexo feminino. 
Figura 1 - Participação quanto ao sexo nos cursos de Computação/Informática.

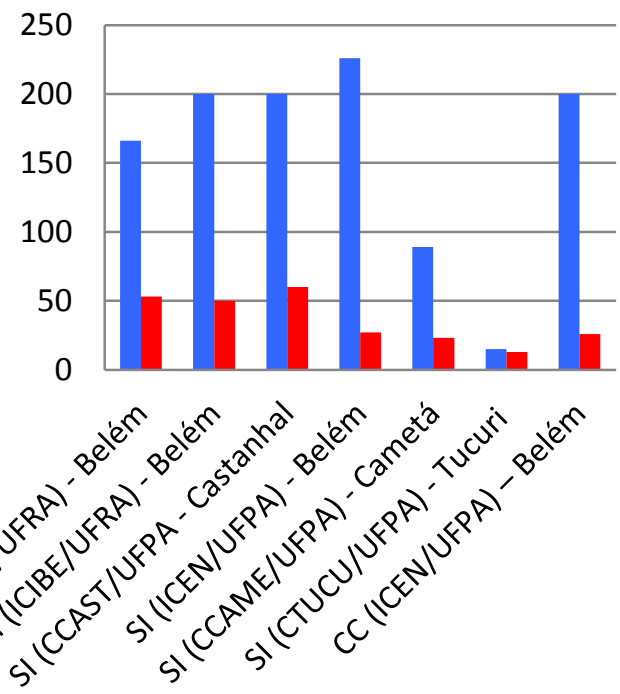

Em seguida, foram utilizados dados dos questionários aplicados aos discentes dos cursos de computação.

$\mathrm{Na}$ primeira etapa, para analisar a participação feminina nos cursos de computação, das instituições públicas situadas em alguns municípios do Estado do Pará, foi aplicado um questionário, composto por vinte questões objetivas e descritivas, coletando respostas de 205 discentes. O questionário foi aplicado no período de 15 a 26 de fevereiro de 2016 e foi elaborado com base no trabalho de Lim (2015), realizado no Massachusetts Institute of Technology MIT.

A partir dos dados coletados, a metodologia consistiu em analisar as respostas de acordo com o sexo dos discentes.
$\mathrm{Na}$ segunda etapa, as questões elaboradas nos questionários representam variáveis, onde as respostas dos(as) discentes são os valores que essas variáveis podem assumir. Por exemplo, para a pergunta "com respeito ao esclarecimento de dúvidas na disciplina de algoritmos e programação, você", a variável é denominada de inibicao e pode assumir os seguintes valores, determinados pela resposta dada pelo discente quanto ao grau de inibição para questionar ou pedir esclarecimentos durante a aula: poucoinibido, inibido, e, muitoinibido. Esse procedimento foi realizado para todas as perguntas do questionário.

No processo de preparação da base de dados obtida a partir dos questionários, foi realizada a formatação e a limpeza dos dados, devido a necessidade da integração destes dados de forma a obter uma base de dados integrada e consistente.

A partir das respostas dos alunos, esta etapa da metodologia envolveu o estudo das associações entre as variáveis selecionadas. Esse estudo permite, por exemplo, identificar se as dificuldades relatadas pelos participantes na disciplina de Algoritmos e Programação (AP) estão associadas ao sexo dos alunos.

Para isso, foram utilizados modelos probabilísticos que permitem realizar inferências sobre as variáveis (respostas) analisadas. Esse estudo utilizou a técnica de 
redes bayesianas, também denominadas de redes causais ou modelos gráficos de dependência probabilística. As redes bayesianas podem ser vistas como modelos que codificam os relacionamentos probabilísticos entre variáveis que representam um determinado domínio. Esses modelos são formados por uma estrutura qualitativa, representando as dependências entre os nós (variáveis) e uma estrutura quantitativa (tabelas de probabilidades condicionais dos nós), quantificando essas dependências em termos probabilísticos (CHEN, 2001). Essas estruturas oferecem uma representação eficiente da distribuição de probabilidades do conjunto de variáveis em estudo - assim, para cada nó, existe uma distribuição de probabilidade local, que depende do estado de seus pais. Para o aprendizado da estrutura da rede bayesiana foi aplicado o algoritmo de busca heurística K2 (COOPER; HERSKOVITS, 1992), tendo como entrada as variáveis identificadas.

A rede bayesiana resultante do processo de busca pela associação entre as variáveis revelou um conjunto de variáveis independentes - questões cujas respostas dos(as) discentes não apresentaram associação. Embora essas questões sejam relevantes e tenham sido analisadas na etapa anterior (de análise dos questionários), essas variáveis foram descartadas nesta etapa do estudo da associação. Em resumo, uma descrição das variáveis onde são encontradas associações, é apresentada na Tabela 1.

Tabela 1 - Variáveis selecionadas.

\begin{tabular}{|l|l|}
\hline Variável & Descrição \\
\hline sexo & Sexo do aluno. \\
\hline curso & $\begin{array}{l}\text { Curso em que o aluno } \\
\text { está matriculado. }\end{array}$ \\
\hline universidade & $\begin{array}{l}\text { Universidade em que } \\
\text { o aluno está } \\
\text { matriculado. }\end{array}$ \\
\hline semestre & $\begin{array}{l}\text { Semestre que o aluno } \\
\text { está cursando. }\end{array}$ \\
\hline dificuldaprendizado & $\begin{array}{l}\text { Resposta do aluno } \\
\text { quanto à dificuldade } \\
\text { de aprendizado na } \\
\text { disciplina de AP. }\end{array}$ \\
\hline inibicao & $\begin{array}{l}\text { Inibição no } \\
\text { esclarecimento de } \\
\text { dúvidas na disciplina } \\
\text { de AP }\end{array}$ \\
\hline A terceira & $\begin{array}{l}\text { Sexo do Professor em } \\
\text { disciplina de AP. }\end{array}$ \\
\hline Aprovacaodiscalgorit & $\begin{array}{l}\text { Situação de } \\
\text { aprovação ou } \\
\text { reprovação na } \\
\text { disciplina de AP. }\end{array}$ \\
\hline
\end{tabular}

metodologia consistiu na realização de entrevistas com oito alunas dos cursos de SI e LC da UFRA, com a finalidade de coletar informações mais detalhadas sobre como essas estudantes veem a participação feminina nesses cursos.

\section{RESULTADOS E DISCUSSÕES}

Os resultados apresentados a seguir seguem as etapas propostas na metodologia: análise dos questionários; análise das 
associações entre as variáveis do estudo; e, entrevistas.

\subsection{Análise dos questionários}

A primeira análise de resultados foi realizada sobre as respostas dos(as) discentes com respeito aos motivos para a seleção do curso, nível de satisfação e desmotivação com o curso, bem como a influência dos grupos de disciplinas que levaram à desmotivação, dificuldade de aprendizado com as disciplinas de algoritmos e programação, sexo dos seus colegas, e, por fim, os motivos para permanecer no curso.

Alguns dos resultados dessa análise, por sexo, são apresentados nas Tabelas 2-6, comentados a seguir.

Dentre os principais motivos para escolher o curso (Tabela 2), independente do sexo do discente, destacam-se o interesse pessoal e a oportunidade de trabalho na área.
Tabela 2 - Motivos para escolher o curso.

\begin{tabular}{|c|c|c|}
\hline Motivo & $\begin{array}{l}\text { Mulheres } \\
\text { (\%) }\end{array}$ & $\begin{array}{l}\text { Homens } \\
(\%)\end{array}$ \\
\hline $\begin{array}{l}\text { Avaliação positiva do } \\
\text { curso (reconhecimento da } \\
\text { comunidade externa) }\end{array}$ & 11,8 & 16,9 \\
\hline $\begin{array}{l}\text { Encorajamento a partir de } \\
\text { um membro da família }\end{array}$ & 15,7 & 11,7 \\
\hline $\begin{array}{l}\text { Experiência positiva em } \\
\text { alguma atividade anterior } \\
\text { à faculdade }\end{array}$ & 15,7 & 24,7 \\
\hline $\begin{array}{l}\text { impacto acadêmico do } \\
\text { curso escolhido }\end{array}$ & 5,9 & 8,4 \\
\hline $\begin{array}{l}\text { Impacto social do curso } \\
\text { escolhido }\end{array}$ & 3,9 & 9,1 \\
\hline Interesse pessoal & 51,0 & 64,9 \\
\hline $\begin{array}{l}\text { Oportunidade de trabalho } \\
\text { na área }\end{array}$ & 51,0 & 42,9 \\
\hline Pura curiosidade & 2,0 & 0,0 \\
\hline $\begin{array}{l}\text { Oferta maior de vagas no } \\
\text { SISU }\end{array}$ & 2,0 & 0,0 \\
\hline $\begin{array}{l}\text { Encorajamento a partir de } \\
\text { um colega de profissão }\end{array}$ & 2,0 & 3,2 \\
\hline Não sei responder & 2,0 & 0,6 \\
\hline Outros & 0,0 & 0,6 \\
\hline $\begin{array}{l}\text { Com respeito ao } \\
\text { com o curso (Tabela 3), } \\
\text { diferença percentual } \\
\text { padrão=2,17). }\end{array}$ & $\begin{array}{l}\text { nível de } \\
\text { não foi en } \\
\text { ignificativa }\end{array}$ & $\begin{array}{l}\text { atisfação } \\
\text { contrada } \\
\text { (desvio }\end{array}$ \\
\hline
\end{tabular}

Tabela 3 - Nível de satisfação com o curso.

\begin{tabular}{|l|l|l|}
\hline Satisfação com o curso & $\begin{array}{l}\text { Mulheres } \\
(\%)\end{array}$ & $\begin{array}{l}\text { Homens } \\
(\%)\end{array}$ \\
\hline Indiferente & 0 & 5,19 \\
\hline Pouco insatisfeito & 5,9 & 7,1 \\
\hline $\begin{array}{l}\text { Insatisfeito ou muito } \\
\text { insatisfeito }\end{array}$ & 5,9 & 9,7 \\
\hline Pouco satisfeito & 19,6 & 13,6 \\
\hline $\begin{array}{l}\text { Satisfeito ou totalmente } \\
\text { satisfeito }\end{array}$ & 68,6 & 64,3 \\
\hline
\end{tabular}


Dentre os entrevistados, 108 discentes responderam que já pensaram em desistir do curso, sendo que, dentre as mulheres, o percentual é superior $(66,7 \%)$ que o dos homens $(48,1 \%)$ (Tabela 4). Dentre os que já pensaram em desistir do curso, quando questionados sobre a influência do grupo de disciplinas, as disciplinas de algoritmos e programação foram apontadas por um percentual maior de mulheres $(37,3 \%)$ (Tabela 5). Dentre os principais motivos para permanecer no curso, o aspecto mais apontado foi o fato de gostar do conteúdo do curso. Entretanto, o percentual de homens que apontam esse aspecto é maior ( $57,8 \%$ contra $45,1 \%)$.

Tabela 4 - Desmotivação/desistência quanto ao curso.

\begin{tabular}{|l|l|l|}
\hline Já pensou em desistir? & $\begin{array}{l}\text { Mulheres } \\
(\%)\end{array}$ & $\begin{array}{l}\text { Homens } \\
(\%)\end{array}$ \\
\hline Sim & 66,7 & 48,1 \\
\hline Não & 27,5 & 44,8 \\
\hline Não sei responder & 5,9 & 7,1 \\
\hline
\end{tabular}

Tabela 5 - Influência do grupo de disciplinas para desmotivação.

\begin{tabular}{|l|l|l|}
\hline Grupo de disciplinas & $\begin{array}{l}\text { Mulheres } \\
(\%)\end{array}$ & $\begin{array}{l}\text { Homens } \\
(\%)\end{array}$ \\
\hline $\begin{array}{l}\text { Disciplinas de algoritmos } \\
\text { e programação }\end{array}$ & 37,3 & 21,4 \\
\hline Disciplinas de Cálculo & 9,8 & 13,0 \\
\hline Disciplinas de humanas & 2,0 & 3,2 \\
\hline Outros & 51,0 & 62,3 \\
\hline
\end{tabular}

Tabela 6 - Motivos para permanecer no curso.

\begin{tabular}{|l|l|l|}
\hline Motivo & $\begin{array}{l}\text { Mulheres } \\
(\%)\end{array}$ & $\begin{array}{l}\text { Homens } \\
(\%)\end{array}$ \\
\hline O incentivo familiar & 25,5 & 14,3 \\
\hline Trabalhos em equipe & 15,7 & 10,4 \\
\hline $\begin{array}{l}\text { Me sinto competente } \\
\text { para a área }\end{array}$ & 27,5 & 29,9 \\
\hline $\begin{array}{l}\text { O incentivo de } \\
\text { Professores/Técnicos }\end{array}$ & 19,6 & 11,7 \\
\hline $\begin{array}{l}\text { O fato de gostar do } \\
\text { conteúdo do curso }\end{array}$ & 45,1 & 57,8 \\
\hline $\begin{array}{l}\text { A influência positiva de } \\
\text { colegas da área }\end{array}$ & 25,5 & 11,7 \\
\hline $\begin{array}{l}\text { Possibilidade de ser bem } \\
\text { remunerado (a) na } \\
\text { carreira }\end{array}$ & 33,3 & 32,5 \\
\hline $\begin{array}{l}\text { Quero mostrar para as } \\
\text { pessoas que sou capaz } \\
\text { de ser bem sucedido }\end{array}$ & 15,7 & 16,2 \\
\hline $\begin{array}{l}\text { Possibilidade de fazer } \\
\text { intercâmbio }\end{array}$ & 5,9 & 18,8 \\
\hline Outros & 2,0 & 0,6 \\
\hline
\end{tabular}

\subsection{Estudo da associação entre variáveis}

Na sequência, com o objetivo de analisar a influência do sexo do discente com respeito às demais variáveis do estudo, foi aplicada a técnica de redes bayesianas. Nesta etapa, foram eliminadas as variáveis onde não foram encontradas associações. A rede bayesiana resultante é apresentada na Figura 2. 
Figura 2 - Rede Bayesiana para as variáveis do estudo.

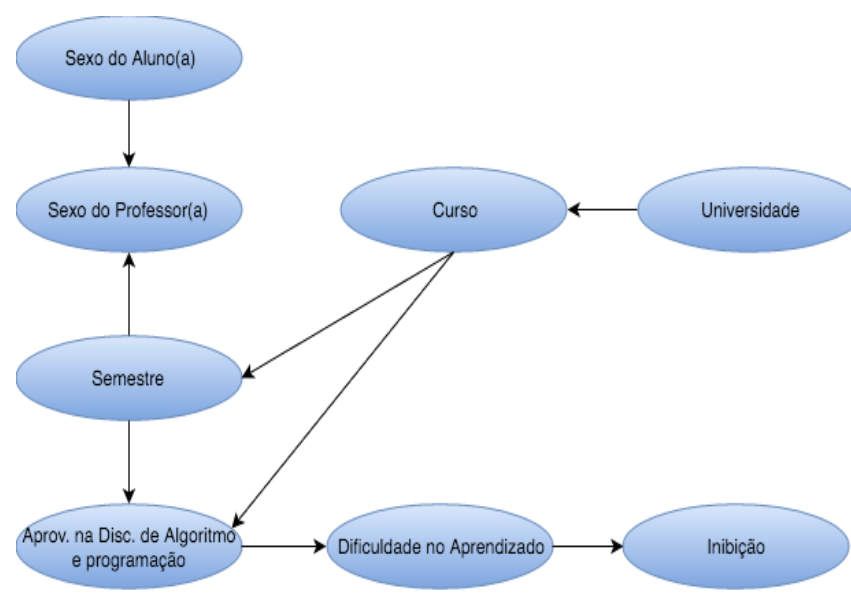

Com respeito ao sexo do professor(a), não foi encontrada associação com a inibição do(a) discente ou dificuldade de aprendizado. Por exemplo, quando a evidência é que o professor é do sexo masculino, a probabilidade do(a) discente se sentir inibido ou muito inibido é de $32,2 \%$ (Figura 3 ) contra $29,9 \%$ quando a evidência é do professor ser do sexo feminino (Figura 4).
Figura 3. Tabelas de probabilidades condicionais dada evidência do docente ser do sexo masculino.

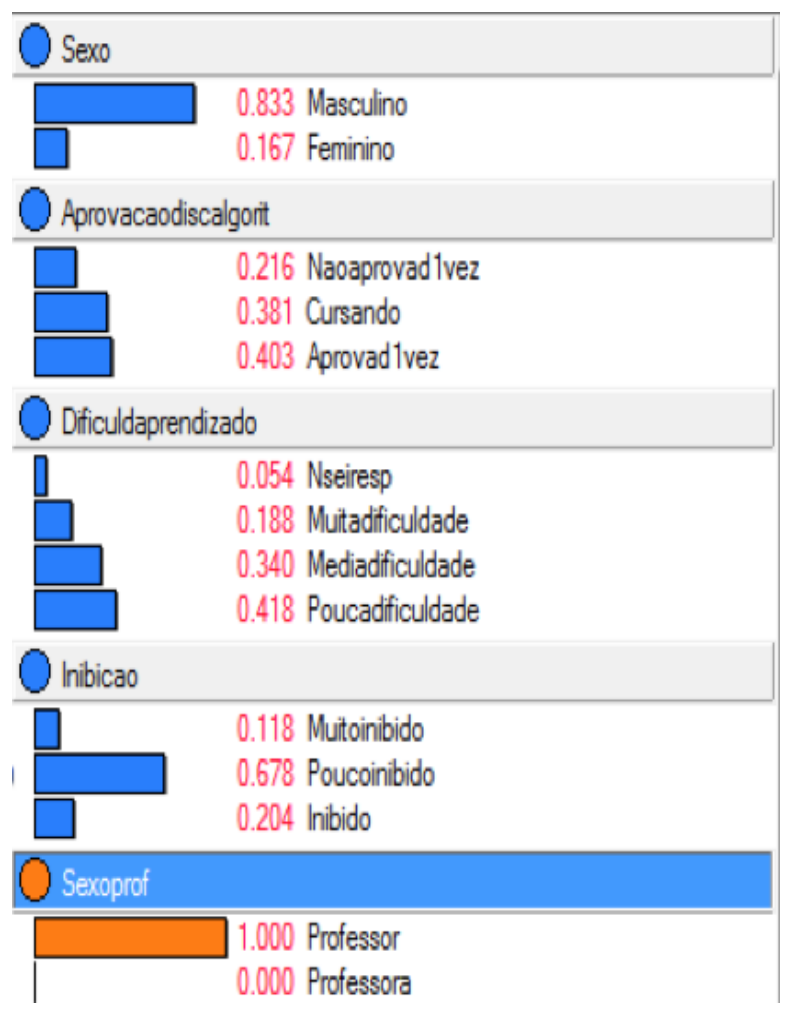

Figura 4 - Tabelas de probabilidades condicionais dada evidência do docente ser do sexo feminino.

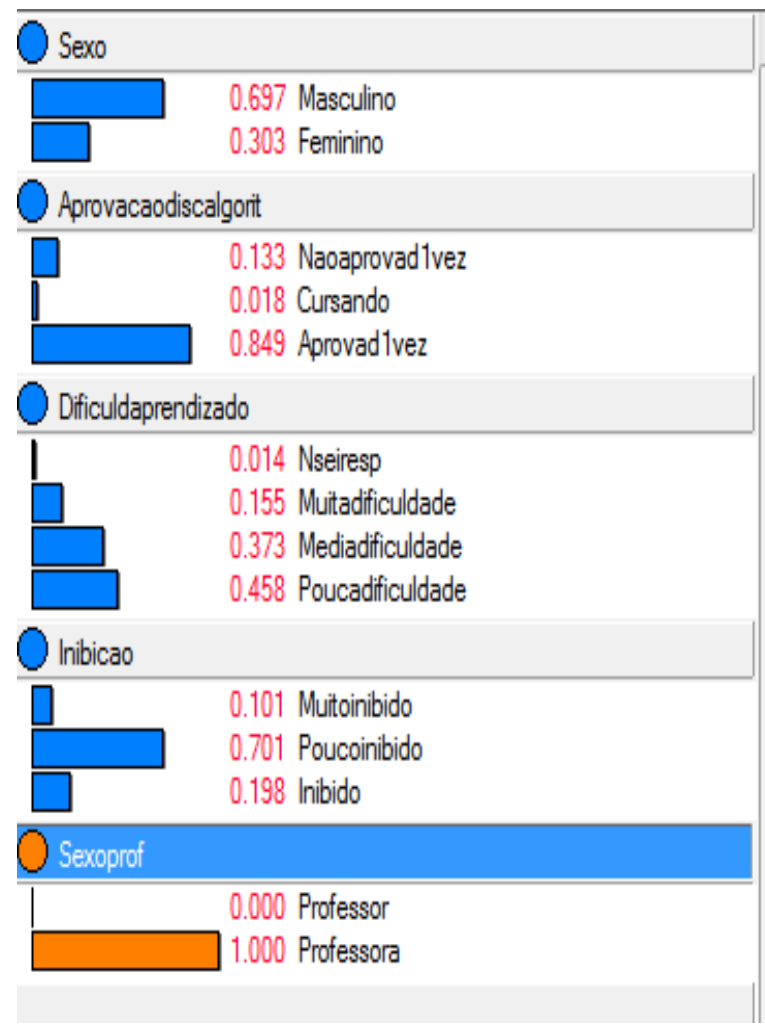


Entretanto, foi encontrada uma associação significativa entre sexo do docente e dificuldade de aprendizado nas disciplinas de AP. Dada a evidência de que o docente seja do sexo masculino e discente do sexo feminino, há uma probabilidade de $35,5 \%$ da aluna relatar pouca dificuldade de aprendizado, contra $47,8 \%$ quando se trata de docente do sexo feminino (Figura 6).

Também foi encontrada associação maior entre a alta inibição das alunas quando se trata de docente do sexo masculino: a probabilidade de relatar sentir-se inibida ou muito inibida é de $36,4 \%$ contra $28,5 \%$, quando se trata de professora (Figura 6).

Figura 5 - Tabelas de probabilidades condicionais (evidência de docente do sexo masculino e discente do sexo feminino).

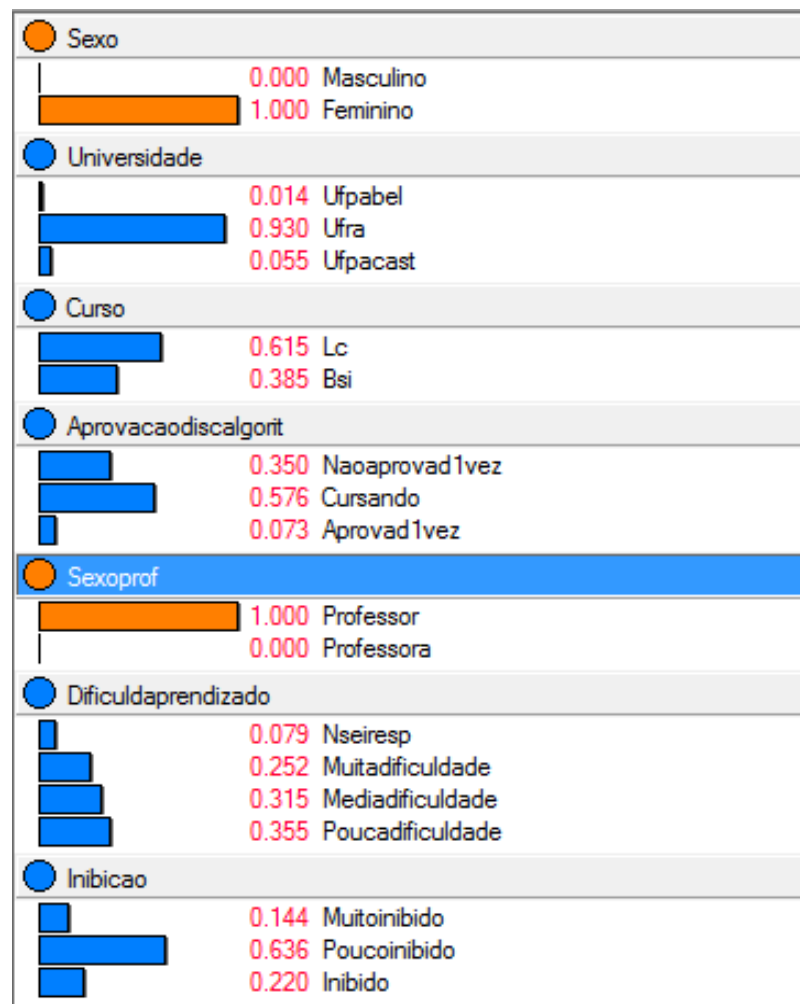

Figura 6 - Tabelas de probabilidades condicionais (evidência de docente do sexo feminino e discente do sexo feminino).

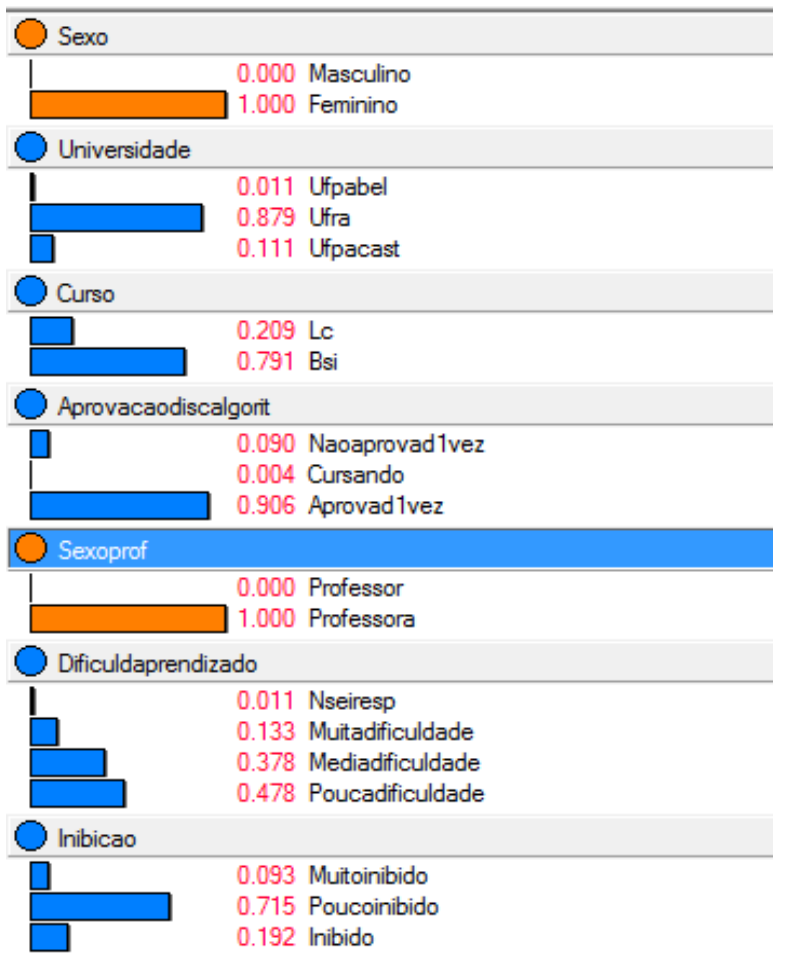

\subsection{Entrevistas}

Na questão de gênero, sabemos que não há campos de atuação onde as mulheres estejam livres da segregação, pois a divisão sexual é a mais persistente e indiscutivelmente a mais profunda no mundo de hoje (EPSTEIN, 2007). É primordial reconstruir os espaços acadêmicos e profissionais, fazendo com que homens e mulheres possam trabalhar e demonstrar suas habilidades de forma igualitária, baseado apenas em sua competência e profissionalismo, melhorando por sua vez a produtividade e desenvolvimento dentro das organizações, instituições e empresas em geral.

Nessa direção, com o objetivo de capturar informações mais detalhadas, foram 
realizadas entrevistas com oito alunas dos cursos de SI e LC da UFRA. Nessas entrevistas, as alunas descreveram suas experiências e dificuldades, dando atenção aos problemas enfrentados em sala de aula.

A partir das entrevistas, metade das entrevistadas confirmaram o fato do número de mulheres em sala de aula ser muito inferior que o número de homens representa um obstáculo em alguma ocasião ao longo do curso. Diferentes alunas relataram:

- "me sentia inibida durante as aulas quando tinha alguma dúvida";

- "sentia muito receio ao manifestar minha opinião ou questionar dentro de sala de aula, me via como uma "estranha" lá dentro".

Quanto à influência do sexo do professor, uma aluna relatou que: "No curso há uma postura de alguns professores, principalmente em relação às meninas, que não respondem e fingem não ouvir as alunas, mas se um homem fala a mesma coisa ele ouve e responde". Percebe-se aqui, que para além da inibição, pode haver um sentimento de menor importância dada às perguntas das alunas - o que pode afetar negativamente a forma como as mulheres se veem no curso.

Também é interessante relatar que a maioria das entrevistadas afirmam que o fato de terem tido professoras mulheres influenciaram de forma positiva na sua vida acadêmica. Além disso, todas as entrevistadas relataram que acreditam que as ações e iniciativas que visam empoderar as mulheres podem promover maior participação das mulheres nos cursos e no mercado de trabalho na área da Computação e Tecnologia, removendo barreiras sociais e culturais que impedem as mulheres de atingir seu potencial nessas áreas. Também, em sua totalidade, reconhecem que um engajamento maior de homens e meninos para construção de novas relações de gênero sem atitudes e comportamentos machistas, pode ter influência positiva na ampliação da participação feminina nesses cursos. Nesse contexto, uma das entrevistadas do curso de LC ressaltou que "é preciso acabar com o preconceito na área - que ainda tem muito" e outra, de SI, apontou que "o empoderamento da Mulher é significativo como fator de sua participação".

A referência de mulheres da computação também é relatado como um aspecto importante, conforme o relato de uma aluna de LC:

"Acredito que ter referência de mulheres que são extraordinárias em nossa área e divulgar que elas existem sim, e ocupam seus cargos com a melhor qualidade possivel é muito importante. Aqui mesmo na Universidade, temos professoras nota mil em conhecimento quando se trata de computação, informática, entre outros. São os nossos, pelo menos o meu, ponto de referência para o 
interesse, desenvolvimento $e$ progresso na minha profissão. "

Para finalizar, em outro relato, uma aluna de SI comenta que:

"Ainda precisamos de muitas mudanças dentro da área de $\mathrm{TI}$, principalmente mudar com esse ambiente machista $e$ as dificuldades que as mulheres passam na área".

Em relação à indagação sobre como deve ser trabalhado e como pode ser a contribuição para o aumento das mulheres na área, uma aluna de LC ressaltou que é preciso:

"Levar mais da nossa área para as mulheres, fornecer cursos, explicações e informações sobre $o$ curso e quais os benefícios do mesmo, além de desfazer esses conceitos $e$ definições que separam áreas masculinas $e$ femininas".

Da mesma forma, uma aluna de SI destacou a importância de "realizar projetos que divulguem o curso $e$ que sejam direcionados para meninas dentro das escolas".

\section{CONSIDERAÇÕES FINAIS}

Através desta pesquisa explanatória, com resultados quantitativos e qualitativos sobre a participação das mulheres na computação, se fez um breve mapeamento em torno dos cursos de computação do estado do Pará. O objetivo foi analisar respostas dos(as) discentes com respeito à diferentes aspectos, sob a ótica do gênero do discente: motivos para a seleção do curso, nível de satisfação e desmotivação com o curso, influência dos grupos de disciplinas que levaram à desmotivação, dificuldade de aprendizado com as disciplinas de algoritmos e programação, motivos para permanecer no curso, dentre outros.

A metodologia envolveu três etapas: análise das respostas dos(as) discentes nos questionários; estudo da associação entre as respostas dos(as) discentes; e, entrevistas com discentes.

Ao identificar tendências atuais sobre o que inspira e incentiva as mulheres a escolher os cursos de TI, este estudo pretende contribuir para a literatura que auxilia na concepção das iniciativas e programas que visam ampliar a participação feminina nesses cursos. Por outro lado, este estudo tem uma limitação quanto ao público investigado - a amostra é pequena e restringe-se aos estudantes dos cursos de $\mathrm{TI}$. Nessa direção, como sugestão para trabalhos posteriores, pretende-se ampliar o público alvo da pesquisa para incluir estudantes do ensino médio, com o objetivo de identificar as razões pelas quais as mulheres não optam pelos cursos de TI.

Além disso, no contexto dos estudos da evasão nos cursos de computação e informática, esta pesquisa reforça a 
importância de inserir as questões de gênero nesses estudos.

Embora o estudo aponte que há pouca influência do sexo do(a) discente nos aspectos de inibição, dificuldade de aprendizado e desmotivação, relatos apontam ainda para a necessidade de iniciativas, programas e ações que incentivam a participação feminina nesses cursos.

\section{REFERÊNCIAS}

CABRAL, C; BAZZO, W. As mulheres nas escolas de engenharia brasileiras: história, educação e futuro. Revista de Ensino de Engenharia, v. 24, n. 1, 2005.

CAMPBELL, L. O.; HERLIHY, C. An analysis of the media's sociocultural influence of women in technology: implications for educators. Society for Information Technology \& Teacher Education International Conference, p. 474-478, 2016.

CHEN, Z. Data Mining and uncertain reasoning: an integrated approach. New York: John Wiley \& Sons, 2001.

COOPER G.F.; HERSKOVITS E. A Bayesian method for the induction of probabilistic networks from data. Machine Learning, v.9, n. 4, p. 309-347, 1992. https://doi.org/10.1007/BF00994110

DOMÍNGUEZ, L. et al. Digital competences' diagnosis in Higher Education: an overview of the differences between the first female and male digital natives students' technological access in Mondragon Unibertsitatea on two years. In: INTED2011 - INTERNATIONAL CONFERENCE ON TECHNOLOGY, EDUCATION AND DEVELOPMENT. Proceedings... Valencia, Espanha, p. 6151-6159, 2011.
EPSTEIN, C. F. Great divides: the cultural, cognitive, and social bases of the global subordination of women. American Sociological Review, v. 72, n. 1, p. 1-22, 2007. https://doi.org/10.1177/0003122407072001 $\underline{01}$

GIL-JUAREZ, A. et al. Brecha digital de género: una revisión y una propuesta. Teoría de la Educación, v. 12, n. 2, p. 18, 2011.

IBGE. Pesquisa Nacional por Amostra de Domicílios. 2014. Disponível em: <http://www.ibge.gov.br/home/estatistica/p opulacao/trabalhoerendimento/pnad2014>.

Acesso em: 12 nov. 2015.

LIM, K. B. Understanding the gender differences in factors affecting the decision to study engineering $t$ MIT. Tese (Doutorado) - Massachusetts Institute of Technology, $2015 . \quad$ Disponível em: $<$ http://dspace.mit.edu/handle/1721.1/9877 9>. Acesso em: 12 nov. 2015.

MACIEL, C.; BIM, S. A. Programa Meninas Digitais - ações para divulgar a computação para meninas do ensino médio. In: COMPUTER ON THE BEACH. Anais... Florianópolis: Universidade do Vale do Itajaí, 2016.

MORAIS, N. S.; RAMOS, F. As questões de género e o uso de tecnologias da informação e comunicação em contexto educacionaluma revisão de literatura. Indagatio Didactica, v. 8, n. 2, 2016.

NUNES, D. J. Educação superior em computação-estatísticas 2012. Sociedade Brasileira de Computação, 2012. Disponível em: <http://www.sbc.org.br/index.php>

NUNES, M. M. et al. Uma iniciativa para atrair as estudantes do ensino médio para a Área de Tecnologia da Informação. In: WORKSHOP DE INFORMÁTICA NA ESCOLA. Anais... 2015. p.

425.

https://doi.org/10.5753/cbie.wie.2015.425 
SABOYA, M. C. L. Alunas de engenharia elétrica e ciência da computação: estudar, inventar, resistir. 2013. Disponível em: <http://www.faceq.edu.br/regs/downloads/ numero05/alunasengenhariaeletricacienciac omputacao.pdf>. Acesso em: 12 nov. 2015.

SBC. Meninas digitais. 2016. Disponível em: <http://www.sbc.org.br/institucional-

3/meninas-digitais>. Acesso em: 12 out. 2016.

SCHWARTZ, J. et al. Mulheres na informática: quais foram as pioneiras? Cadernos Pagu. v. 27, n. 1, p. 255-278, 2006. https://doi.org/10.1590/S0104-

$\underline{83332006000200010}$

UNIÃO EUROPEIA. Comissão Europeia. Faltam mulheres no sector europeu das tecnologias da informação e das comunicações. 2008. Disponível em: <http://europa.eu/rapid/pressReleasesActio $\mathrm{n}$.

do? reference $=\mid P / 08 / 392 \&$ format=PDF\&age $=1 \&$ language $=$ PT\&guiLanguage $=e n>$. Acesso em: 05 nov. 2016. 